\title{
Parikh's Theorem in Commutative Kleene Algebra
}

\author{
Mark W. Hopkins \\ Adaptive Micro Systems, Inc. \\ 7840 North 86th St. \\ Milwaukee, Wisconsin 53224, USA \\ mwh@ams-i.com
}

\author{
Dexter C. Kozen \\ Department of Computer Science \\ Cornell University \\ Ithaca, New York 14853-7501, USA \\ kozen@cs.cornell.edu
}

\begin{abstract}
Parikh's Theorem says that the commutative image of every context free language is the commutative image of some regular set. Pilling has shown that this theorem is essentially a statement about least solutions of polynomial inequalities. We prove the following general theorem of commutative Kleene algebra, of which Parikh's and Pilling's theorems are special cases: Every finite system of polynomial inequalities $f_{i}\left(x_{1}, \ldots, x_{n}\right) \leq x_{i}, 1 \leq i \leq n$, over a commutative Kleene algebra $K$ has a unique least solution in $K^{n}$; moreover, the components of the solution are given by polynomials in the coefficients of the $f_{i}$. We also give a closed-form solution in terms of the Jacobian matrix of the system.
\end{abstract}

\section{Introduction}

Parikh's theorem [9] says that every context-free language is "letter-equivalent" to a regular set; formally, the commutative image of any context-free language is also the commutative image of some regular set, where the commutative image of a set $A$ of strings over the finite alphabet $\left\{a_{1}, \ldots, a_{k}\right\}$ is the set of $k$-tuples

$$
\left\{\left(\# a_{1}(x), \ldots, \# a_{k}(x)\right) \in \mathbb{N}^{k} \mid x \in A\right\} \quad \subseteq \mathbb{N}^{k},
$$

where $\# a_{i}(x)$ is the number of occurrences of $a_{i}$ in $x$. The $k$-tuples in $\mathbb{N}^{k}$ are often called Parikh vectors. For example, the context-free language $\left\{a^{n} b^{n} \mid n \geq 0\right\}$ is letterequivalent to the regular set $(a b)^{*}$; these two sets have a common commutative image $\{(n, n) \mid n \geq 0\}$.

The usual combinatorial proofs of Parikh's theorem involve an induction on parse trees of context-free grammars. In this paper we prove the following general theorem of commutative Kleene algebra, of which Parikh's theorem is a special case:
Theorem 1.1 Every system of inequalities

$$
f_{i}\left(x_{1}, \ldots, x_{n}\right) \leq x_{i}, \quad 1 \leq i \leq n,
$$

where the $f_{i}$ are polynomials in $K\left[x_{1}, \ldots, x_{n}\right]$ over a commutative Kleene algebra $K$, has a unique least solution in $K^{n}$; moreover, the components of the solution are given by polynomials in the coefficients of the $f_{i}$.

We might take the statement of Theorem 1.1 as a definition of algebraic closure in Kleene algebra, in which case the theorem says that any commutative Kleene algebra is algebraically closed.

Pilling [10] proves Theorem 1.1 in the special case of the commutative Kleene algebra $\operatorname{Reg}\left(\mathbb{N}^{k}\right)$, the algebra of regular sets of Parikh vectors, and argues that this is the essential content of Parikh's theorem. Indeed, context-free grammars are just systems of set inequalities, and the context-free languages they generate are the minimal solutions. For example, the context-free grammar $S \rightarrow a S b \mid \epsilon$ is essentially the system consisting of the single inequality $a x b+1 \leq x$ whose least solution in $2^{\{a, b\}^{*}}$ is the context-free language $\left\{a^{n} b^{n} \mid n \geq 0\right\}$. Under the assumption of commutativity, the inequality can be rewritten $a b x+1 \leq x$, whose least solution is the regular set $(a b)^{*}$. For a somewhat more difficult example, the context-free grammar $S \rightarrow[S]|S S| \epsilon$ generating the set of balanced strings of parentheses is essentially the system consisting of the single inequality $[x]+x x+1 \leq x$. Under the assumption of commutativity, the inequality can be rewritten [ ] $x+x^{2}+1 \leq x$, whose least solution is the regular set $\left([\mathrm{]})^{*}\right.$.

Using results of $[4$, Lemma 7.1, p. 35] and [7, Section 2.3, p. 198] one can generalize Pilling's proof to any *-continuous Kleene algebra. However, the proof makes essential use of various infinitary properties such as the continuity of regular operators and the fact that $a^{*}$ is the supremum of the $a^{n}, n \geq 0$.

Kuich [8] also gives a generalization of Parikh's theorem that holds for any commutative idempotent $\omega$-continuous semiring. Kuich's result implies Pilling's, since $\operatorname{Reg}\left(\mathbb{N}^{k}\right)$ 
is embedded in the commutative idempotent $\omega$-continuous semiring $2^{\mathbb{N}^{k}}$. Conversely, since every commutative idempotent $\omega$-continuous semiring is a commutative Kleene algebra under the usual definition of the * operator

$$
a^{*}=\sum_{n \geq 0} a^{n},
$$

Pilling's result, suitably generalized to *-continuous Kleene algebras, would imply Kuich's. But again, these proofs depend on the strong infinitary properties of *-continuous algebras.

Our result is a generalization of these results in that it holds in all commutative Kleene algebras. The main difference here is that Kleene algebra as defined in [5] has a finitary algebraic axiomatization consisting of finitely many equations and equational implications. Thus one might say that we are replacing the analytic arguments of Pilling and Kuich with algebraic arguments. The fact that we cannot argue combinatorially in the model $\operatorname{Reg}\left(\mathbb{N}^{k}\right)$ or use the infinitary properties of *-continuous algebras makes the proof more difficult, but also makes the result considerably stronger.

The situation is analogous to the fundamental theorem of algebra, which states that the complex numbers $\mathbb{C}$ are algebraically closed. The most common proof of this theorem, originally due to Gauss, depends on the analytic structure of $\mathbb{C}$ and uses second-order arguments (see e.g. [12]). However, one can give a first-order, purely algebraic proof of the more general result that if $R$ is any real closed field (such as $\mathbb{R}$ or $\mathbb{A}$, the real algebraic numbers), then $R[i]$ is algebraically closed (see e.g. [11]). Like the fundamental theorem of algebra, our result also deals with solutions of polynomial systems, and our proof replaces arguments referring to the analytic or second-order structure of $\operatorname{Reg}\left(\mathbb{N}^{k}\right)$, embodied in the *-continuity axiom, with first-order equational arguments referring only to the finitary algebraic structure of commutative Kleene algebras.

Our development involves the definition of differential operators $\frac{\partial}{\partial x}$ on commutative Kleene algebras of polynomials and a version of Taylor's theorem:

$$
f(x+d)=f(x)+f^{\prime}(x+d) \cdot d .
$$

Differential operators allow us to define the Jacobian matrix of a system of inequalities, which we use to give a closed form solution.

In Section 2 we review the definitions of Kleene algebra and commutative Kleene algebra. In Section 3 we discuss polynomials over a commutative Kleene algebra, define differential operators on a commutative Kleene algebra of polynomials, and develop some basic properties, culminating in a version of Taylor's theorem. In Section 4 we prove Theorem 1.1. In Section 5 we give a closed form solution in terms of the Jacobian matrix of a system of inequalities.

\section{Commutative Kleene Algebra}

Kleene algebra is the algebra of regular expressions [3, 1]. The axiomatization we adopt here is from [5]. A Kleene algebra is an algebraic structure $\left(K,+, \cdot{ }^{*}, 0,1\right)$ that is an idempotent semiring under $+, \cdot, 0,1$ satisfying

$$
\begin{aligned}
1+p p^{*} & =p^{*} \\
1+p^{*} p & =p^{*} \\
q+p r \leq r & \rightarrow p^{*} q \leq r \\
q+r p \leq r & \rightarrow q p^{*} \leq r
\end{aligned}
$$

where $\leq$ refers to the natural partial order on $K$ :

$$
p \leq q \stackrel{\text { def }}{\Longleftrightarrow} p+q=q .
$$

The operation + gives the supremum with respect to the natural order $\leq$. Instead of (4) and (5), we might take the equivalent axioms

$$
\begin{aligned}
& p r \leq r \rightarrow p^{*} r \leq r \\
& r p \leq r \rightarrow r p^{*} \leq r .
\end{aligned}
$$

These axioms say essentially that * behaves like the Kleene asterate operator of formal language theory or the reflexive transitive closure operator of relational algebra.

A Kleene algebra is *-continuous if it satisfies the additional infinitary axiom

$$
p q^{*} r=\sup _{n \geq 0} p q^{n} r
$$

where the supremum on the right-hand side is with respect to the natural order $\leq$. A Kleene algebra or *-continuous Kleene algebra is commutative if it satisfies the additional axiom $p q=q p$.

Kleene algebras play a prominent role in dynamic logic and other program logics. Standard models include the family of regular sets over a finite alphabet; the family of binary relations on a set; and the family of $n \times n$ matrices over another Kleene algebra. Other more unusual interpretations include the min,+ algebra used in shortest path algorithms and models consisting of convex polyhedra used in computational geometry [2]. All naturally occurring models are *continuous. Commutativity assumptions also arise in practice [6].

The following are some typical identities of Kleene algebra:

$$
\begin{aligned}
\left(p^{*} q\right)^{*} p^{*} & =(p+q)^{*} \\
p(q p)^{*} & =(p q)^{*} p \\
p^{*} & =(p p)^{*}(1+p)
\end{aligned}
$$


All the operators are monotone with respect to $\leq$. In other words, if $p \leq q$, then $p r \leq q r, r p \leq r q, p+r \leq q+r$, and $p^{*} \leq q^{*}$ for any $r$.

The following is a theorem of commutative Kleene algebra that does not hold in Kleene algebra in general:

$$
(p+q)^{*}=p^{*} q^{*} \text {. }
$$

Using this, one can prove a normal form theorem that says that every expression is equivalent to a sum $y_{1}+\cdots+y_{n}$, where each $y_{i}$ is a product of atomic symbols and expressions of the form $\left(a_{1} \cdots a_{k}\right)^{*}$, where the $a_{i}$ are atomic symbols. For example,

$$
\left(\left((a b)^{*} c\right)^{*}+d\right)^{*}=d^{*}+(a b)^{*} c^{*} c d^{*} .
$$

This normal form was observed by Pilling [10] in the context of $\operatorname{Reg}\left(\mathbb{N}^{k}\right)$, but using (11) it is easily shown to hold in all commutative Kleene algebras.

The equational theory of Kleene algebras and *continuous Kleene algebras coincide [5], but their Horn theories do not; indeed, the Horn theory of $*$-continuous Kleene algebras is $\Pi_{1}^{1}$-complete [7]. bra.

See [5] for a more thorough introduction to Kleene alge-

\section{Polynomials and Differential Operators}

\subsection{Polynomials over a commutative Kleene algebra}

If $K$ is a commutative Kleene algebra, we denote by $K[\mathbf{x}]$ the commutative Kleene algebra of polynomials in indeterminates $\mathbf{x}$ over $K$. These are very much like polynomials over a ring or field. We can think of a polynomial as a regular expression over $K$ and $\mathbf{x}$ reduced modulo the axioms of commutative Kleene algebra and the diagram of $K$ (the set of ground identities that hold in $K$ ). Typical examples of polynomials are

$$
\begin{gathered}
(a x+b y)^{*} \\
1+\left(a x^{*} b^{*}\right)^{*}+b x+c y \\
a+x y(b x y)^{*},
\end{gathered}
$$

where $x, y$ are indeterminates and $a, b, c \in K$.

Formally, $K[\mathbf{x}]$ is defined to be the direct sum (coproduct) of $K$ with the free commutative Kleene algebra on generators $\mathbf{x}$ in the category of commutative Kleene algebras. The most significant property of polynomials is that any pair of maps $h, h^{\prime}$, where $h: K \rightarrow L$ is a Kleene algebra homomorphism and $h^{\prime}: \mathbf{x} \rightarrow L$ is a set function, extend simultaneously and uniquely to a Kleene algebra homomorphism $\widehat{h}: K[\mathbf{x}] \rightarrow L$. When $h$ is the identity on $K$, the map $\widehat{h}$ is just polynomial evaluation; intuitively, applying $\widehat{h}$ can be regarded as substituting the values $h^{\prime}(x)$ for the indeterminates $x \in \mathbf{x}$ and then evaluating the resulting expression.

If $\mathbf{x}=x_{1}, \ldots, x_{n}$ and $\mathbf{a}=a_{1}, \ldots, a_{n}$, we write $f(\mathbf{a})$ or $\left.f(\mathbf{x})\right|_{\mathbf{x}=\mathbf{a}}$ for the value of $f$ evaluated at $x_{i} \mapsto a_{i}, 1 \leq i \leq$ $n$.

\subsection{Differential Operators}

A map $D: K \rightarrow K$ on a commutative Kleene algebra $K$ is called a differential operator if for all $x, y \in K$,

$$
\begin{aligned}
D(x+y) & =D x+D y \\
D(x y) & =x D y+y D x \\
D\left(x^{*}\right) & =x^{*} D x \\
D 0 & =D 1=0 .
\end{aligned}
$$

For example, in $\operatorname{Reg}\left(\mathbb{N}^{k}\right)$, for every $1 \leq i \leq k$, the map

$$
\begin{gathered}
A \mapsto \quad\left\{\left(a_{1}, \ldots, a_{i-1}, a_{i}-1, a_{i+1}, \ldots, a_{k}\right) \mid\right. \\
\left.\left(a_{1}, \ldots, a_{k}\right) \in A, a_{i}>0\right\}
\end{gathered}
$$

is a differential operator.

Theorem 3.1 Any differential operator $D: K \rightarrow K$ and set function $D: \mathbf{x} \rightarrow K$ have a unique joint extension to a differential operator $D: K[\mathbf{x}] \rightarrow K[\mathbf{x}]$.

Proof. The given maps $D$ can be extended by induction to $D: K[\mathbf{x}] \rightarrow K[\mathbf{x}]$ using (12); but we must take care that the extended $D$ is well-defined on equivalence classes modulo the axioms of commutative Kleene algebra and the diagram of $K$. That the extended $D$ respects the diagram of $K$ follows from the fact that the given $D: K \rightarrow K$ is a differential operator on $K$. To prove that $D$ respects the commutative Kleene algebra axioms requires a case for each axiom. We argue the cases $a^{*}=1+a a^{*}$ and $a b \leq$ $b \rightarrow a^{*} b \leq b$ explicitly.

For the case $a^{*}=1+a a^{*}$,

$$
\begin{aligned}
D\left(1+a a^{*}\right) & =D 1+D\left(a a^{*}\right) \\
& =0+a D\left(a^{*}\right)+a^{*} D a \\
& =a a^{*} D a+a^{*} D a \\
& =a^{*} D a \\
& =D\left(a^{*}\right) .
\end{aligned}
$$

For the case $a b \leq b \rightarrow a^{*} b \leq b$, suppose $a b \leq b$. By the induction hypothesis, $D(a b) \leq D b$, and we wish to show that $D\left(a^{*} b\right) \leq D b$. From $D(a b) \leq D b$ we have that $a D b+$ $b D a \leq D b$, thus by (6) we have that $a^{*} D b \leq D b$, and by (4) we have that $a^{*} b D a \leq D b$. Therefore

$$
\begin{aligned}
D\left(a^{*} b\right) & =a^{*} D b+b D\left(a^{*}\right) \\
& =a^{*} D b+b a^{*} D a \\
& \leq D b .
\end{aligned}
$$


In particular, for $x \in \mathbf{x}$, we define a certain differential operator $\frac{\partial}{\partial x}: K[\mathbf{x}] \rightarrow K[\mathbf{x}]$ as follows. The value of $\frac{\partial}{\partial x}$ applied to $f \in K[\mathbf{x}]$ is denoted $\frac{\partial f}{\partial x}$ or $\frac{\partial f}{\partial x}(\mathbf{x})$. We define $\frac{\partial}{\partial x}$ to be the unique differential operator such that $\frac{\partial x}{\partial x}=1$, $\frac{\partial y}{\partial x}=0$ for $y \in \mathbf{x}-\{x\}$, and $\frac{\partial a}{\partial x}=0$ for $a \in K$.

For univariate polynomials $f, e \in K[x]$, we sometimes write $f^{\prime}$ for $\frac{\partial f}{\partial x}$ and $\frac{\partial f}{\partial x}(e)$ or $f^{\prime}(e)$ for the result of evaluating the polynomial $\frac{\partial f}{\partial x}$ at $x \mapsto e$.

For example, if $f(x)=a x b+x^{2}+1$, then $f^{\prime}(x)=$ $a b+x$.

Note that $\frac{\partial}{\partial x}(f(e))$ and $\frac{\partial f}{\partial x}(e)$ are different in general. The former refers to the result of evaluating $f(x)$ at $x \mapsto e$ first, then applying the differential operator $\frac{\partial}{\partial x}$ to $f(e)$; whereas the latter refers to the result of applying the differential operator $\frac{\partial}{\partial x}$ to $f(x)$ first, then evaluating the resulting polynomial $\frac{\partial f}{\partial x}(x)$ at $x \mapsto e$. These two expressions are related by the chain rule:

Theorem 3.2 (chain rule) For $f, e \in K[x]$,

$$
\frac{\partial}{\partial x}(f(e))=\frac{\partial f}{\partial x}(e) \cdot \frac{\partial e}{\partial x},
$$

or in more conventional notation,

$$
f(e(x))^{\prime}=f^{\prime}(e(x)) \cdot e^{\prime}(x) .
$$

Proof. This is a straightforward induction on the structure of $f$. We argue the cases $f=g h$ and $f=g^{*}$ explicitly.

$$
\begin{aligned}
(g(e) h(e))^{\prime} & =g(e) h(e)^{\prime}+h(e) g(e)^{\prime} \\
& =g(e) h^{\prime}(e) e^{\prime}+h(e) g^{\prime}(e) e^{\prime} \\
& =\left(g(e) h^{\prime}(e)+h(e) g^{\prime}(e)\right) e^{\prime} \\
& =\left(g h^{\prime}+h g^{\prime}\right)(e) e^{\prime} \\
& =(g h)^{\prime}(e) e^{\prime} . \\
\left(g(e)^{*}\right)^{\prime} & =g(e)^{*} g(e)^{\prime} \\
& =g(e)^{*} g^{\prime}(e) e^{\prime} \\
& =\left(g^{*} g^{\prime}(e) e^{\prime}\right. \\
& =\left(g^{*}\right)^{\prime}(e) e^{\prime} .
\end{aligned}
$$

For example, if $f(x)=a x+x^{*}+1$, then

$$
\begin{aligned}
f^{\prime}(x) & =a+x^{*} \\
f(f(x)) & =a\left(a x+x^{*}+1\right)+\left(a x+x^{*}+1\right)^{*}+1 \\
& =a^{2} x+a x^{*}+(a x)^{*} x^{*}+1 \\
f(f(x))^{\prime} & =\left(a^{2} x+a x^{*}+(a x)^{*} x^{*}+1\right)^{\prime} \\
& =a^{2}+(a+1) x^{*}(a x)^{*} \\
f^{\prime}(f(x)) f^{\prime}(x) & =\left(a+\left(a x+x^{*}+1\right)^{*}\right)\left(a+x^{*}\right) \\
& =a^{2}+(a+1) x^{*}(a x)^{*} .
\end{aligned}
$$

We also have the following version of Taylor's theorem in commutative Kleene algebra.
Theorem 3.3 (Taylor's theorem) For $f, d \in K[x]$,

$$
f(x+d)=f(x)+f^{\prime}(x+d) \cdot d .
$$

In particular, evaluating at $x \mapsto 0$,

$$
f(d)=f(0)+f^{\prime}(d) \cdot d .
$$

Proof. This is again a straightforward induction on the structure of $f$. As before, we argue the cases $f=g h$ and $f=g^{*}$ explicitly. For the case $f=g h$,

$$
\begin{aligned}
& g h(x+d) \\
&= g(x+d) h(x+d) \\
&= g(x+d) h(x)+g(x+d) h^{\prime}(x+d) d \\
&= g(x) h(x)+g^{\prime}(x+d) h(x) d \\
& \quad+g(x+d) h^{\prime}(x+d) d,
\end{aligned}
$$

and by symmetry,

$$
\begin{aligned}
& g h(x+d) \\
& =\quad g(x) h(x)+g^{\prime}(x+d) h(x+d) d \\
& \quad+g(x) h^{\prime}(x+d) d,
\end{aligned}
$$

therefore by monotonicity,

$$
\begin{aligned}
g h(x+d) & \\
= & g(x) h(x)+g^{\prime}(x+d) h(x) d+g(x+d) h^{\prime}(x+d) d \\
& \quad+g^{\prime}(x+d) h(x+d) d+g(x) h^{\prime}(x+d) d \\
= & g(x) h(x)+g(x+d) h^{\prime}(x+d) d \\
\quad & \quad g^{\prime}(x+d) h(x+d) d \\
= & g h(x)+(g h)^{\prime}(x+d) d .
\end{aligned}
$$

For the case $f=g^{*}$,

$$
\begin{aligned}
g & (x+d)^{*} \\
& =\left(g(x)+g^{\prime}(x+d) d\right)^{*} \\
& =g(x)^{*}\left(g^{\prime}(x+d) d\right)^{*} \quad \text { by }(11) \\
& =g(x)^{*}+g(x)^{*} g^{\prime}(x+d) d\left(g^{\prime}(x+d) d\right)^{*} \\
& =g(x)^{*}+g^{\prime}(x+d) d g(x)^{*}\left(g^{\prime}(x+d) d\right)^{*} \\
& =g(x)^{*}+g^{\prime}(x+d) d g(x+d)^{*} \\
& =g(x)^{*}+g(x+d)^{*} g^{\prime}(x+d) d \\
& =g(x)^{*}+\left(g^{*}\right)^{\prime}(x+d) d .
\end{aligned}
$$

Continuing with the example $f(x)=a x+x^{*}+1$ above,

$$
\begin{aligned}
f(x+d) & =a(x+d)+(x+d)^{*}+1 \\
& =a x+a d+x^{*} d^{*}+1 \\
f^{\prime}(x+d) & =a+(x+d)^{*} \\
& =a+x^{*} d^{*} \\
f(x)+f^{\prime}(x+d) d & =a x+x^{*}+1+a d+x^{*} d^{*} d \\
& =a x+a d+x^{*} d^{*}+1 .
\end{aligned}
$$


We often wish to differentiate simultaneously with respect to a sequence of indeterminates $\mathbf{y}=y_{1}, \ldots, y_{k}$. We define an operator $\frac{\partial}{\partial \mathbf{y}}$ that when applied to an element $f \in K[\mathbf{x}]$ produces a row vector of length $k$ whose $i^{\text {th }}$ component is $\frac{\partial f}{\partial y_{i}}$. More generally, $\frac{\partial}{\partial \mathbf{y}}$ applied to a column vector consisting of $m$ elements $f_{1}, \ldots, f_{m} \in K[\mathbf{x}]$ produces an $m \times k$ matrix whose $i, j^{\text {th }}$ element is $\frac{\partial f_{i}}{\partial y_{j}}$.

By iterating Theorem 3.3, one can show that for $f \in$ $K[\mathbf{x}]$ and $\mathbf{e}=e_{1}, \ldots, e_{n}$,

$$
\begin{aligned}
f(\mathbf{e}) & =f(0, \ldots, 0)+\frac{\partial f}{\partial x_{1}}(\mathbf{e}) e_{1}+\cdots+\frac{\partial f}{\partial x_{n}}(\mathbf{e}) e_{n} \\
& =f(\mathbf{0})+\frac{\partial f}{\partial \mathbf{x}}(\mathbf{e}) \cdot \mathbf{e}
\end{aligned}
$$

where $\cdot$ denotes dot product of vectors. The same holds for a column vector $\mathbf{f}=f_{1}, \ldots, f_{m}$ of elements of $K[\mathbf{x}]$; here $\frac{\partial \mathbf{f}}{\partial \mathbf{x}}(\mathbf{x})$ is an $m \times n$ matrix whose $i, j^{\text {th }}$ element is $\frac{\partial f_{i}}{\partial x_{j}}$, and

$$
\mathbf{f}(\mathbf{e})=\mathbf{f}(\mathbf{0})+\frac{\partial \mathbf{f}}{\partial \mathbf{x}}(\mathbf{e}) \cdot \mathbf{e},
$$

where in this case - denotes matrix-vector multiplication. The $m \times n$ matrix $\frac{\partial \mathbf{f}}{\partial \mathbf{x}}(\mathbf{x})$ is called the Jacobian of $\mathbf{f}$.

We also have the following vector-vector and matrixvector versions of the chain rule, Theorem 3.2:

$$
\begin{aligned}
\frac{\partial}{\partial z}(f(\mathbf{e})) & =\frac{\partial f}{\partial x_{1}}(\mathbf{e}) \frac{\partial e_{1}}{\partial z}+\cdots+\frac{\partial f}{\partial x_{n}}(\mathbf{e}) \frac{\partial e_{n}}{\partial z} \\
& =\frac{\partial f}{\partial \mathbf{x}}(\mathbf{e}) \cdot \frac{\partial \mathbf{e}}{\partial z} \\
\frac{\partial}{\partial z}(\mathbf{f}(\mathbf{e})) & =\frac{\partial \mathbf{f}}{\partial \mathbf{x}}(\mathbf{e}) \cdot \frac{\partial \mathbf{e}}{\partial z} .
\end{aligned}
$$

The proof of these propositions is a straightforward generalization of the proof of Theorem 3.2.

\section{A Generalization of Parikh's Theorem}

In this section we prove Theorem 1.1. We first prove the result for $n=1$, then extend it to arbitrary $n$. Many arguments in this section are inspired by those of Pilling [10] (see also [1]) but generalized to apply to arbitrary commutative Kleene algebras.

Theorem 4.1 Let $K$ be a commutative Kleene algebra and let $f(x) \in K[x]$. The unique least solution of the inequality $f(x) \leq x$ is

$$
f^{\prime}(f(0))^{*} \cdot f(0)
$$

Moreover, this holds uniformly over all homomorphic images of $K$.

For example, the context-free language $A=\left\{a^{n} b^{n}\right.$ $n \geq 0\}$ is generated by the grammar $S \rightarrow a S b \mid \epsilon$, which translates to the one-dimensional system $a x b+1 \leq x$. Letting $f(x)=a x b+1$, we get $f^{\prime}(x)=a b$ and $f(0)=1$, thus (14) gives $(a b)^{*}$. This is a regular expression describing a regular set letter-equivalent to $A$.
Proof. First we argue that (14) is a solution to $f(x) \leq x$. It follows by a straightforward inductive argument that for any polynomial $h(x)$,

$$
a c \leq b c \rightarrow h(a) c \leq h(b) c .
$$

Applying this with $b=f(0), c=f^{\prime}(b)^{*}$, and $a=b c$,

$$
\begin{array}{rlrl}
f(a) & =f(b c) & \\
& =f(0)+f^{\prime}(b c) b c & & \text { by Theorem } 3.3 \\
& =b+f^{\prime}(b c) b c & \\
& \leq b+f^{\prime}(b) b c & & \text { by }(15) \\
& =b+f^{\prime}(b) f^{\prime}(b)^{*} b & & \\
& =f^{\prime}(b)^{*} b & & \text { by Kleene algebra } \\
& =a . &
\end{array}
$$

Now we show that (14) is the least solution. Suppose $y$ is any solution; thus $f(y) \leq y$. We wish to show that

$$
f^{\prime}(f(0))^{*} f(0) \leq y
$$

By (4), it suffices to show

$$
f(0)+f^{\prime}(f(0)) \cdot y \leq y .
$$

But by monotonicity, $f(0) \leq f(y) \leq y$, and

$$
\begin{aligned}
& f(0)+f^{\prime}(f(0)) \cdot y \\
& \leq f(0)+f^{\prime}(y) \cdot y \quad \text { by monotonicity } \\
& =f(y) \quad \text { by Theorem } 3.3 \\
& \leq y \text {. }
\end{aligned}
$$

The expression (14) gives the least solution of $f(x) \leq x$ uniformly over all homomorphic images of $K$ because the axioms of Kleene algebra used in the proof hold universally under any interpretation.

The uniformity condition of Theorem 4.1 may seem obvious, but it is actually a rather subtle point. The issue is that equations are preserved under homomorphisms, but in general Horn formulas (equational implications) are not. The homomorphic image $h(e)$ of a solution $e$ of an inequality $f(x) \leq x$ is a solution of the homomorphic image of the inequality, because the inequality is equivalent to an equation $f(x)+x=x$; but that $h(e)$ is the least solution does not follow from the fact that $e$ is least, since this property requires a Horn formula.

Proof of Theorem 1.1. We iterate the one-dimensional solution as follows. Consider the two-dimensional system

$$
\begin{aligned}
& f(x, y) \leq x \\
& g(x, y) \leq y
\end{aligned}
$$


Viewing $K[x, y]$ as $K[x][y]$, first compute the least solution to the one-dimensional system $g(x, y) \leq y$ in $K[x]$; call it $h(x)$. Then compute the least solution $a$ of $f(x, h(x)) \leq x$ in $K$.

We claim that $(a, h(a))$ is the desired least solution to (16) in $K^{2}$. Surely $f(a, h(a)) \leq a$ by the one-dimensional argument. Moreover, by the uniformity observation, we also have $g(a, h(a)) \leq h(a)$, since it is the image of $g(x, h(x)) \leq h(x)$ under the evaluation homomorphism $x \mapsto a$.

To show $(a, h(a))$ is the least solution, suppose $(b, c)$ is any other solution. Then $f(b, c) \leq b$ and $g(b, c) \leq c$. Using the uniformity observation with the evaluation morphism $x \mapsto b$, we have that $h(b)$ is the least solution of $g(b, y) \leq y$. Then $h(b) \leq c$. But by monotonicity, $f(b, h(b)) \leq f(b, c) \leq b$. Since $a$ is the least solution to $f(x, h(x)) \leq x$, we have that $a \leq b$. Again by monotonicity, $h(a) \leq h(b) \leq c$. Thus $(a, h(a)) \leq(b, c)$.

By iterating this process inductively, we can obtain the existence of a solution to any $n \times n$ system.

\section{A Closed Form Solution}

The iterated construction of the previous section does not give a symmetric closed-form expression for any dimension greater than one. In this section we provide a symmetric closed-form solution.

Let $K$ be a commutative Kleene algebra and consider an $n \times n$ system

$$
\mathbf{f}(\mathbf{x}) \leq \mathbf{x}
$$

where $\mathbf{x}=x_{1}, \ldots, x_{n}$ and $\mathbf{f}=\mathbf{f}(\mathbf{x})=f_{1}(\mathbf{x}), \ldots, f_{n}(\mathbf{x}) \in$ $K[\mathbf{x}]$. Let $\frac{\partial \mathbf{f}}{\partial \mathbf{x}}$ be the Jacobian of the system (17) as defined in Section 3.2. Define

$$
\begin{aligned}
\mathbf{a}_{0} & \stackrel{\text { def }}{=} \\
\mathbf{a}_{k+1} & \stackrel{\mathbf{f}(\mathbf{0})}{=} \frac{\partial \mathbf{f}}{\partial \mathbf{x}}\left(\mathbf{a}_{k}\right)^{*} \mathbf{a}_{k} .
\end{aligned}
$$

Theorem 5.1 For sufficiently large finite $N$, the $n$-vector $\mathbf{a}_{N}$ is the least solution to (17). Moreover, this solution is uniform over all homomorphic images of $K$.

Below we will derive an explicit single-exponential bound on $N$ as a function of $n$.

Proof. We will prove the first statement of the theorem; the uniformity property will follow by the same considerations as in the proof of Theorem 4.1. The proof proceeds by induction on $n$. The basis $n=1$ was given in Theorem 4.1.

Now suppose $n \geq 2$. Partition $n$ as $m+(n-m)$ where $1 \leq m<n$. For an $n$-vector $\mathbf{b}=b_{1}, \ldots, b_{n}$, write

$$
\begin{array}{ll}
\mathbf{b}^{\mathbf{Q}} \stackrel{\text { def }}{=} b_{1}, \ldots, b_{m} \\
\mathbf{b}^{\mathbf{\square}} \stackrel{\text { def }}{=} b_{m+1}, \ldots, b_{n},
\end{array}
$$

and for an $n \times n$ matrix $M$, write

$$
M^{\boxplus}, \quad M^{\boxplus}, \quad M^{\boxplus}, \quad M^{\boxplus}
$$

for the upper left $m \times m$, upper right $m \times(n-m)$, lower left $(n-m) \times m$, and lower right $(n-m) \times(n-m)$ submatrices of $M$, respectively. To simplify notation, define

$$
\begin{array}{lll}
\mathbf{y} \stackrel{\text { def }}{=} \mathbf{x}, & \mathbf{g} \stackrel{\text { def }}{=} \mathbf{f}^{\mathbf{\square}}, \\
\mathbf{z} \stackrel{\text { def }}{=} \mathbf{q}, & \mathbf{h} \stackrel{\text { def }}{=} \mathbf{f}^{\mathbf{q}} .
\end{array}
$$

In this notation, we can rewrite (17) as

$$
\begin{aligned}
& \mathbf{g}(\mathbf{y}, \mathbf{z}) \leq \mathbf{y} \\
& \mathbf{h}(\mathbf{y}, \mathbf{z}) \leq \mathbf{z} .
\end{aligned}
$$

Also,

$$
\begin{array}{ll}
\frac{\partial \mathbf{g}}{\partial \mathbf{y}}=\frac{\partial \mathbf{f}}{\partial \mathbf{x}} \mathbf{\boxplus}, & \frac{\partial \mathbf{g}}{\partial \mathbf{z}}=\frac{\partial \mathbf{f}}{\partial \mathbf{x}} \mathbf{\boxplus}, \\
\frac{\partial \mathbf{h}}{\partial \mathbf{y}}=\frac{\partial \mathbf{f}}{\partial \mathbf{x}} \mathbf{\boxplus}, & \frac{\partial \mathbf{h}}{\partial \mathbf{z}}=\frac{\partial \mathbf{f}}{\partial \mathbf{x}} \boldsymbol{\boxplus} .
\end{array}
$$

Now define

$$
\begin{aligned}
\mathbf{c}_{0}(\mathbf{y}) \stackrel{\text { def }}{=} \mathbf{h}(\mathbf{y}, \mathbf{0}) \\
\mathbf{c}_{k+1}(\mathbf{y}) \stackrel{\text { def }}{=} \frac{\partial \mathbf{h}}{\partial \mathbf{z}}\left(\mathbf{y}, \mathbf{c}_{k}(\mathbf{y})\right)^{*} \mathbf{c}_{k}(\mathbf{y})
\end{aligned}
$$

By the induction hypthesis, there exists a $P$ such that $\mathbf{c}_{P}(\mathbf{y})$ is the least solution to the system

$$
\mathbf{h}(\mathbf{y}, \mathbf{z}) \leq \mathbf{z}
$$

uniformly in $\mathbf{y}$. Define

$$
\begin{aligned}
& \widehat{\mathbf{g}}(\mathbf{y}) \stackrel{\text { def }}{=} \\
& \mathbf{g}\left(\mathbf{y}, \mathbf{c}_{P}(\mathbf{y})\right) \\
& \mathbf{b}_{0} \stackrel{\text { def }}{=} \widehat{\mathbf{g}}(\mathbf{0}) \\
& \mathbf{b}_{k+1} \stackrel{\text { def }}{=} \frac{\partial \widehat{\mathbf{g}}}{\partial \mathbf{y}}\left(\mathbf{b}_{k}\right)^{*} \mathbf{b}_{k} .
\end{aligned}
$$

Again by the induction hypthesis, there exists an $M$ such that $\mathbf{b}_{M}$ is the least solution to the system

$$
\widehat{\mathbf{g}}(\mathbf{y}) \leq \mathbf{y} .
$$

By the uniformity of the solutions, we have that

$$
\begin{aligned}
& \mathbf{g}\left(\mathbf{b}_{M}, \mathbf{c}_{P}\left(\mathbf{b}_{M}\right)\right) \leq \mathbf{b}_{M} \\
& \mathbf{h}\left(\mathbf{b}_{M}, \mathbf{c}_{P}\left(\mathbf{b}_{M}\right)\right) \leq \mathbf{c}_{P}\left(\mathbf{b}_{M}\right),
\end{aligned}
$$

and $\mathbf{b}_{M}, \mathbf{c}_{P}\left(\mathbf{b}_{M}\right)$ is the least solution to (17). Moreover, this is the least solution uniformly over all homomorphic images.

Our task now is to show that for sufficiently large $N$,

$$
\mathbf{b}_{M}=\mathbf{a}_{N}^{\mathbf{Q}}, \quad \mathbf{c}_{P}\left(\mathbf{b}_{M}\right)=\mathbf{a}_{N}^{\mathbf{Q}} .
$$


The inequalities $\geq$ follow from the fact that if $\mathbf{u}$ is any solution to (17), then $\mathbf{a}_{k} \leq \mathbf{u}$ for all $k$. This can be shown by induction on $k$. Certainly

$$
\mathbf{a}_{0}=\mathbf{f}(\mathbf{0}) \leq \mathbf{f}(\mathbf{u}) \leq \mathbf{u}
$$

and by (13),

$$
\frac{\partial \mathbf{f}}{\partial \mathbf{x}}(\mathbf{u}) \mathbf{u} \leq \mathbf{u}
$$

from which it follows that

$$
\begin{aligned}
\mathbf{a}_{k+1} & =\frac{\partial \mathbf{f}}{\partial \mathbf{x}}\left(\mathbf{a}_{k}\right)^{*} \mathbf{a}_{k} \\
& \leq \frac{\partial \mathbf{f}}{\partial \mathbf{x}}(\mathbf{u})^{*} \mathbf{u} \\
& \leq \mathbf{u}
\end{aligned}
$$

Now we establish a series of inequalities from which the forward inequalities $\leq$ of (19) will follow. First,

$$
\mathbf{a}_{k} \leq \frac{\partial \mathbf{f}}{\partial \mathbf{x}}\left(\mathbf{a}_{k}\right)^{*} \mathbf{a}_{k}=\mathbf{a}_{k+1}
$$

thus $\mathbf{a}_{i} \leq \mathbf{a}_{j}, i \leq j$, and similarly for $\mathbf{b}_{i}$ and $\mathbf{c}_{i}$.

Now we show that

$$
\mathbf{g}\left(\mathbf{a}_{k}\right) \leq \mathbf{a}_{k+1}^{\mathbf{Q}} \quad \mathbf{h}\left(\mathbf{a}_{k}\right) \leq \mathbf{a}_{k+1}^{\nabla}
$$

By (13),

$$
\begin{aligned}
\mathbf{g}\left(\mathbf{a}_{k}\right) & =\mathbf{g}(\mathbf{0})+\frac{\partial \mathbf{g}}{\partial \mathbf{x}}\left(\mathbf{a}_{k}\right) \mathbf{a}_{k} \\
& =\mathbf{f}(\mathbf{0})^{\mathbf{Q}}+\frac{\partial \mathbf{f}}{\partial \mathbf{x}}\left(\mathbf{a}_{k}\right)^{\mathbf{\Xi}_{k}} \\
& =\mathbf{a}_{0}^{\mathbf{G}}+\left(\frac{\partial \mathbf{f}}{\partial \mathbf{x}}\left(\mathbf{a}_{k}\right) \mathbf{a}_{k}\right)^{\mathbf{\square}} \\
& \leq\left(\mathbf{a}_{k}+\frac{\partial \mathbf{f}}{\partial \mathbf{x}}\left(\mathbf{a}_{k}\right)^{*} \mathbf{a}_{k}\right)^{\mathbf{\square}} \\
& =\mathbf{a}_{k+1} .
\end{aligned}
$$

The second inequality of (20) follows from a similar argument.

Now we show by induction on $j$ that

$$
\mathbf{c}_{j}\left(\mathbf{a}_{k}^{\mathbf{Q}}\right) \leq \mathbf{a}_{j+k+1}^{\mathbf{Q}}
$$

For the basis,

$$
\begin{aligned}
\mathbf{c}_{0}\left(\mathbf{a}_{k}^{\mathbf{G}}\right) & =\mathbf{h}\left(\mathbf{a}_{k}^{\mathbf{Q}}, \mathbf{0}\right) \\
& \leq \mathbf{h}\left(\mathbf{a}_{k}\right) \\
& \leq \mathbf{a}_{k+1} .
\end{aligned}
$$

For the induction step,

$$
\begin{aligned}
& \mathbf{c}_{j+1}\left(\mathbf{a}_{k}^{\nabla}\right)=\frac{\partial \mathbf{h}}{\partial \mathbf{z}}\left(\mathbf{a}_{k}^{\nabla}, \mathbf{c}_{j}\left(\mathbf{a}_{k}^{\nabla}\right)\right)^{*} \mathbf{c}_{j}\left(\mathbf{a}_{k}^{\nabla}\right) \\
& \leq \frac{\partial \mathbf{h}}{\partial \mathbf{z}}\left(\mathbf{a}_{k}^{\mathbf{Q}}, \mathbf{a}_{j+k+1}^{\mathbf{Z}}\right)^{*} \mathbf{a}_{j+k+1}^{\mathbf{Z}} \\
& \leq \frac{\partial \mathbf{h}}{\partial \mathbf{z}}\left(\mathbf{a}_{j+k+1}\right) * \mathbf{a}_{j+k+1}^{\mathbf{Q}} \\
& =\left(\frac{\partial \mathbf{f}}{\partial \mathbf{x}}\left(\mathbf{a}_{j+k+1}\right)^{\mathbf{⿴}}\right)^{*} \mathbf{a}_{j+k+1}^{\mathbf{B}} \\
& \leq\left(\frac{\partial \mathbf{f}}{\partial \mathbf{x}}\left(\mathbf{a}_{j+k+1}\right)^{*}\right)^{\mathbf{Q}_{j+k+1}} \\
& \leq\left(\frac{\partial \mathbf{f}}{\partial \mathbf{x}}\left(\mathbf{a}_{j+k+1}\right)^{*} \mathbf{a}_{j+k+1}\right)^{\mathbf{Q}} \\
& =\mathbf{a}_{j+k+2}^{\square} \text {. }
\end{aligned}
$$

Now we show by induction on $k$ that when $m=n-1$, that is, for $|z|=1$,

$$
\frac{\partial \mathbf{c}_{k+1}}{\partial \mathbf{y}}(\mathbf{y}) \leq \frac{\partial \mathbf{h}}{\partial \mathbf{z}}\left(\mathbf{y}, \mathbf{c}_{k}(\mathbf{y})\right)^{*} \frac{\partial \mathbf{h}}{\partial \mathbf{y}}\left(\mathbf{y}, \mathbf{c}_{k}(\mathbf{y})\right) .
$$

The corresponding result for $m<n-1$ would require some specialized notation even to state. The proof for $m=n-1$ is considerably simpler, so we henceforth restrict ourselves to that case.

First we note that

$$
\frac{\partial \mathbf{c}_{0}}{\partial \mathbf{y}}(\mathbf{y})=\frac{\partial}{\partial \mathbf{y}}(\mathbf{h}(\mathbf{y}, \mathbf{0}))=\frac{\partial \mathbf{h}}{\partial \mathbf{y}}(\mathbf{y}, \mathbf{0})
$$

Then

$$
\begin{aligned}
\frac{\partial \mathbf{c}_{k+1}}{\partial \mathbf{y}}(\mathbf{y}) & \\
= & \frac{\partial}{\partial \mathbf{y}}\left(\frac{\partial \mathbf{h}}{\partial \mathbf{z}}\left(\mathbf{y}, \mathbf{c}_{k}(\mathbf{y})\right)^{*} \mathbf{c}_{k}(\mathbf{y})\right) \\
= & \mathbf{c}_{k}(\mathbf{y}) \frac{\partial}{\partial \mathbf{y}}\left(\frac{\partial \mathbf{h}}{\partial \mathbf{z}}\left(\mathbf{y}, \mathbf{c}_{k}(\mathbf{y})\right)^{*}\right) \\
& +\frac{\partial \mathbf{h}}{\partial \mathbf{z}}\left(\mathbf{y}, \mathbf{c}_{k}(\mathbf{y})\right)^{*} \frac{\partial \mathbf{c}_{k}}{\partial \mathbf{y}}(\mathbf{y}) \\
= & \mathbf{c}_{k}(\mathbf{y}) \frac{\partial \mathbf{h}}{\partial \mathbf{z}}\left(\mathbf{y}, \mathbf{c}_{k}(\mathbf{y})\right)^{*} \frac{\partial}{\partial \mathbf{y}}\left(\frac{\partial \mathbf{h}}{\partial \mathbf{z}}\left(\mathbf{y}, \mathbf{c}_{k}(\mathbf{y})\right)\right) \\
& +\frac{\partial \mathbf{h}}{\partial \mathbf{z}}\left(\mathbf{y}, \mathbf{c}_{k}(\mathbf{y})\right)^{*} \frac{\partial \mathbf{c}_{k}}{\partial \mathbf{y}}(\mathbf{y}) \\
= & \frac{\partial \mathbf{h}}{\partial \mathbf{z}}\left(\mathbf{y}, \mathbf{c}_{k}(\mathbf{y})\right)^{*} \mathbf{c}_{k}(\mathbf{y}) \frac{\partial}{\partial \mathbf{y}}\left(\frac{\partial \mathbf{h}}{\partial \mathbf{z}}\left(\mathbf{y}, \mathbf{c}_{k}(\mathbf{y})\right)\right) \\
& +\frac{\partial \mathbf{h}}{\partial \mathbf{z}}\left(\mathbf{y}, \mathbf{c}_{k}(\mathbf{y})\right)^{*} \frac{\partial \mathbf{c}_{k}}{\partial \mathbf{y}}(\mathbf{y}) \\
\leq & \frac{\partial \mathbf{h}}{\partial \mathbf{z}}\left(\mathbf{y}, \mathbf{c}_{k}(\mathbf{y})\right)^{*} \frac{\partial}{\partial \mathbf{y}}\left(\frac{\partial \mathbf{h}}{\partial \mathbf{z}}\left(\mathbf{y}, \mathbf{c}_{k}(\mathbf{y})\right) \mathbf{c}_{k}(\mathbf{y})\right) \\
& +\frac{\partial \mathbf{h}}{\partial \mathbf{z}}\left(\mathbf{y}, \mathbf{c}_{k}(\mathbf{y})\right)^{*} \frac{\partial \mathbf{c}_{k}}{\partial \mathbf{y}}(\mathbf{y}) \\
\leq & \frac{\partial \mathbf{h}}{\partial \mathbf{z}}\left(\mathbf{y}, \mathbf{c}_{k}(\mathbf{y})\right)^{*} \frac{\partial}{\partial \mathbf{y}}\left(\mathbf{h}\left(\mathbf{y}, \mathbf{c}_{k}(\mathbf{y})\right)\right) \\
& +\frac{\partial \mathbf{h}}{\partial \mathbf{z}}\left(\mathbf{y}, \mathbf{c}_{k}(\mathbf{y})\right)^{*} \frac{\partial \mathbf{c}_{k}}{\partial \mathbf{y}}(\mathbf{y}) \\
= & \frac{\partial \mathbf{h}}{\partial \mathbf{z}}\left(\mathbf{y}, \mathbf{c}_{k}(\mathbf{y})\right)^{*}\left(\frac{\partial \mathbf{h}}{\partial \mathbf{y}}\left(\mathbf{y}, \mathbf{c}_{k}(\mathbf{y})\right)\right. \\
& \left.+\frac{\partial \mathbf{h}}{\partial \mathbf{z}}\left(\mathbf{y}, \mathbf{c}_{k}(\mathbf{y})\right) \frac{\partial \mathbf{c}_{k}}{\partial \mathbf{y}}(\mathbf{y})\right) \\
& +\frac{\partial \mathbf{h}}{\partial \mathbf{z}}\left(\mathbf{y}, \mathbf{c}_{k}(\mathbf{y})\right)^{*} \frac{\partial \mathbf{c}_{k}}{\partial \mathbf{y}}(\mathbf{y}) \\
= & \frac{\partial \mathbf{h}}{\partial \mathbf{z}}\left(\mathbf{y}, \mathbf{c}_{k}(\mathbf{y})\right)^{*}\left(\frac{\partial \mathbf{h}}{\partial \mathbf{y}}\left(\mathbf{y}, \mathbf{c}_{k}(\mathbf{y})\right)+\frac{\partial \mathbf{c}_{k}}{\partial \mathbf{y}}(\mathbf{y})\right),
\end{aligned}
$$

so it suffices to show

$$
\frac{\partial \mathbf{c}_{k}}{\partial \mathbf{y}}(\mathbf{y}) \leq \frac{\partial \mathbf{h}}{\partial \mathbf{z}}\left(\mathbf{y}, \mathbf{c}_{k}(\mathbf{y})\right)^{*} \frac{\partial \mathbf{h}}{\partial \mathbf{y}}\left(\mathbf{y}, \mathbf{c}_{k}(\mathbf{y})\right)
$$


For $k=0$, this is immediate from (23). For $k>0$, this follows from (24) and the induction hypothesis.

Now we show by induction on $k$ that

$$
\mathbf{b}_{k} \leq \mathbf{a}_{(k+1)(P+2)}^{\mathbf{Z}}
$$

For the basis,

$$
\begin{aligned}
\mathbf{b}_{0} & =\widehat{\mathbf{g}}(\mathbf{0}) \\
& =\mathbf{g}\left(\mathbf{0}, \mathbf{c}_{P}(\mathbf{0})\right) \\
& \leq \mathbf{g}\left(\mathbf{0}, \mathbf{c}_{P}\left(\mathbf{a}_{0}^{\mathbf{0}}\right)\right) \\
& \leq \mathbf{g}\left(\mathbf{0}, \mathbf{a}_{P+1}\right) \\
& \leq \mathbf{g}\left(\mathbf{a}_{P+1}\right) \\
& \leq \mathbf{a}_{P+2} .
\end{aligned}
$$

For the induction step,

$$
\begin{aligned}
& \mathbf{b}_{k+1} \\
& =\frac{\partial \widehat{\mathbf{g}}}{\partial \mathbf{y}}\left(\mathbf{b}_{k}\right)^{*} \mathbf{b}_{k} \\
& =\left.\frac{\partial}{\partial \mathbf{y}}\left(\mathbf{g}\left(\mathbf{y}, \mathbf{c}_{P}(\mathbf{y})\right)\right)^{*} \mathbf{y}\right|_{\mathbf{y}=\mathbf{b}_{k}} \\
& =\left(\frac{\partial \mathbf{g}}{\partial \mathbf{y}}\left(\mathbf{y}, \mathbf{c}_{P}(\mathbf{y})\right)\right. \\
& \left.+\frac{\partial \mathbf{g}}{\partial \mathbf{z}}\left(\mathbf{y}, \mathbf{c}_{P}(\mathbf{y})\right) \frac{\partial \mathbf{c}_{P}}{\partial \mathbf{y}}(\mathbf{y})\right)\left.^{*} \mathbf{y}\right|_{\mathbf{y}=\mathbf{b}_{k}} \\
& =\left(\frac{\partial \mathbf{g}}{\partial \mathbf{y}}\left(\mathbf{b}_{k}, \mathbf{c}_{P}\left(\mathbf{b}_{k}\right)\right)\right. \\
& \left.+\frac{\partial \mathbf{g}}{\partial \mathbf{z}}\left(\mathbf{b}_{k}, \mathbf{c}_{P}\left(\mathbf{b}_{k}\right)\right) \frac{\partial \mathbf{c}_{P}}{\partial \mathbf{y}}\left(\mathbf{b}_{k}\right)\right)^{*} \mathbf{b}_{k} \\
& \leq\left(\frac{\partial \mathbf{g}}{\partial \mathbf{y}}\left(\mathbf{b}_{k}, \mathbf{c}_{P}\left(\mathbf{b}_{k}\right)\right)\right. \\
& +\frac{\partial \mathbf{g}}{\partial \mathbf{z}}\left(\mathbf{b}_{k}, \mathbf{c}_{P}\left(\mathbf{b}_{k}\right)\right) \frac{\partial \mathbf{h}}{\partial \mathbf{z}}\left(\mathbf{b}_{k}, \mathbf{c}_{P-1}\left(\mathbf{b}_{k}\right)\right)^{*} \\
& \left.\cdot \frac{\partial \mathbf{h}}{\partial \mathbf{y}}\left(\mathbf{b}_{k}, \mathbf{c}_{P-1}\left(\mathbf{b}_{k}\right)\right)\right)^{*} \mathbf{b}_{k} \\
& \leq\left(\frac{\partial \mathbf{g}}{\partial \mathbf{y}}\left(\mathbf{b}_{k}, \mathbf{c}_{P}\left(\mathbf{b}_{k}\right)\right)\right. \\
& +\frac{\partial \mathbf{g}}{\partial \mathbf{z}}\left(\mathbf{b}_{k}, \mathbf{c}_{P}\left(\mathbf{b}_{k}\right)\right) \frac{\partial \mathbf{h}}{\partial \mathbf{z}}\left(\mathbf{b}_{k}, \mathbf{c}_{P}\left(\mathbf{b}_{k}\right)\right)^{*} \\
& \left.\cdot \frac{\partial \mathbf{h}}{\partial \mathbf{y}}\left(\mathbf{b}_{k}, \mathbf{c}_{P}\left(\mathbf{b}_{k}\right)\right)\right)^{*} \mathbf{b}_{k} \\
& =\left(\frac{\partial \mathbf{f}}{\partial \mathbf{x}}\left(\mathbf{b}_{k}, \mathbf{c}_{P}\left(\mathbf{b}_{k}\right)\right)^{\boldsymbol{⿴ 囗 十}}\right. \\
& \left.+\frac{\partial \mathbf{f}}{\partial \mathbf{x}}\left(\mathbf{b}_{k}, \mathbf{c}_{P}\left(\mathbf{b}_{k}\right)\right)^{\mathbf{⿴ 囗 十 ~}_{\frac{\partial \mathbf{f}}{\partial \mathbf{x}}}} \mathbf{b}_{k}, \mathbf{c}_{P}\left(\mathbf{b}_{k}\right)\right)^{\mathbf{⿴}_{*}} \\
& \left.\cdot \frac{\partial \mathbf{f}}{\partial \mathbf{x}}\left(\mathbf{b}_{k}, \mathbf{c}_{P}\left(\mathbf{b}_{k}\right)\right)^{\mathbf{⿴}}\right)^{*} \mathbf{b}_{k} \\
& \leq \frac{\partial \mathbf{f}}{\partial \mathbf{x}}\left(\mathbf{b}_{k}, \mathbf{c}_{P}\left(\mathbf{b}_{k}\right)\right) * \mathbf{H}_{\mathbf{b}_{k}} \\
& \leq \frac{\partial \mathbf{f}}{\partial \mathbf{x}}\left(\mathbf{a}_{(k+1)(P+2)}^{\mathbf{D}}, \mathbf{c}_{P}\left(\mathbf{a}_{(k+1)(P+2)}^{\mathbf{D}}\right)\right) * \mathbf{Q}_{\mathbf{a}_{(k+1)(P+2)}}^{\mathbf{D}} \\
& \leq \frac{\partial \mathbf{f}}{\partial \mathbf{x}}\left(\mathbf{a}_{(k+1)(P+2)}^{\mathbf{Q}}, \mathbf{a}_{(k+2)(P+2)-1}^{\mathbf{Q}} * \mathbf{⿴ 囗 十}_{\mathbf{a}_{(k+1)(P+2)}}^{\mathbf{Q}_{(2)}}\right. \\
& \leq \frac{\partial \mathbf{f}}{\partial \mathbf{x}}\left(\mathbf{a}_{(k+2)(P+2)-1}\right) * \mathbf{\boxplus}_{\mathbf{a}_{(k+2)(P+2)-1}} \\
& \leq\left(\frac{\partial \mathbf{f}}{\partial \mathbf{x}}\left(\mathbf{a}_{(k+2)(P+2)-1}\right)^{*} \mathbf{a}_{(k+2)(P+2)-1}\right)^{\mathbf{\square}} \\
& =\mathbf{a}_{(k+2)(P+2)}^{\mathbf{D}} \text {. }
\end{aligned}
$$

It follows from (21) and (25) that

$$
\begin{aligned}
\mathbf{b}_{M} & \leq \mathbf{a}_{(M+1)(P+2)}, \\
\mathbf{c}_{P}\left(\mathbf{b}_{M}\right) & \leq \mathbf{a}_{(M+2)(P+2)-1} .
\end{aligned}
$$

Taking $m=n-1$, Theorem 4.1 says that $P=1$ suffices. Thus the $N$ in the statement of the theorem is bounded by $(M+2)(P+2)-1=3 M+5$. This gives the following recurrence for $N$ as a function of $n$ :

$$
\begin{aligned}
N(1) & =1 \\
N(n+1) & =3 N(n)+5
\end{aligned}
$$

with solution $N(n)=\left(7 \cdot 3^{n}-5\right) / 2$.

\section{Acknowledgements}

We thank the anonymous referees for their valuable criticism. The support of the National Science Foundation under grant CCR-9708915 is gratefully acknowledged.

\section{References}

[1] J. H. Conway. Regular Algebra and Finite Machines. Chapman and Hall, London, U.K., 1971.

[2] K. Iwano and K. Steiglitz. A semiring on convex polygons and zero-sum cycle problems. SIAM J. Comput., 19(5):883$901,1990$.

[3] S. C. Kleene. Representation of events in nerve nets and finite automata. In C. E. Shannon and J. McCarthy, editors, Automata Studies, pages 3-41. Princeton University Press, Princeton, N.J., 1956.

[4] D. Kozen. The Design and Analysis of Algorithms. SpringerVerlag, New York, 1991.

[5] D. Kozen. A completeness theorem for Kleene algebras and the algebra of regular events. Infor. and Comput., 110(2):366-390, May 1994.

[6] D. Kozen. Kleene algebra with tests. Transactions on Programming Languages and Systems, pages 427-443, May 1997.

[7] D. Kozen. On the complexity of reasoning in Kleene algebra. In Proc. 12th Symp. Logic in Comput. Sci., pages 195-202, Los Alamitos, Ca., June 1997. IEEE.

[8] W. Kuich. The Kleene and Parikh theorem in complete semirings. In T. Ottmann, editor, Proc. 14th Colloq. Automata, Languages, and Programming, volume 267 of Lecture Notes in Computer Science, pages 212-225, New York, 1987. EATCS, Springer-Verlag.

[9] R. J. Parikh. On context-free languages. J. Assoc. Comput. Mach., 13(4):570-581, 1966.

[10] D. L. Pilling. Commutative regular equations and Parikh's theorem. J. London Math. Soc., 6(4):663-666, June 1973.

[11] I. Stewart. Galois Theory. Chapman and Hall, London, 1973.

[12] B. L. van der Waerden. Algebra, volume 1. Frederick Ungar, 1970. 